\title{
Artificial Neural Network based Defect Detection of Welds in TOFD Technique
}

\author{
S.Lalithakumari \\ Sathyabama University \\ Chennai - 600119 \\ India
}

\author{
B.Sheelarani \\ Sathyabama University \\ Chennai - 600119 \\ India
}

\author{
B. Venkatraman \\ IGCAR, Kalpakkam \\ India
}

\begin{abstract}
Abstract: Time of Flight Diffraction Technique is one of the NDE methods, used in weld inspection to identify the weld defects. The classification of defects using the TOFD technique depends on the knowledge and experience of the operator. The classification reliability of defects detected by this technique can be improved by applying the Artificial Neural Network. In this work, four austenitic stainless steel weldments with defects viz, Lack of Fusion, Lack of Penetration, Slag, Porosity and one with out any Defect were fabricated. TOFD experiment is conducted on these weldments. Discrete wavelet transform based denoising methods were applied to denoise the resultant A scan signals. Time scale features are extracted from the denoised signals. A multi layer feed forward network with Resilient Back Propagation algorithm has been applied for classification of the signals. The number of hidden layers in the network are increased from 0 to 6 . Various performance functions are also employed to achieve a better classification efficiency. The results are promising to proceed the automatic defect classification by TOFD technique.
\end{abstract}

\section{Keywords}

Resilient Back Propagation algorithm, time scale features, classification accuracy

\section{INTRODUCTION}

Many Non Destructive Techniques are adopted to ensure the quality of welding. The TOFD technique is being widely used for automatic weld inspection.[1]. The two ultrasonic angle probes are used in the TOFD experiment, meant for transmission and reception. The lateral wave travels along the surface, where as the back wall echo reflects the bottom surface of the test object and reach to the receiver. The other two signals, upper flaw tip diffracted signal and lower flaw tip diffracted signal appear due to in homogeneity [2]. Austenitic stainless steel material is used for manufacturing the safety vessels of prototype fast breeder reactor (PFBR).In this paper, TOFD technique is applied on austenitic stainless steel weld defects and neural network based algorithm is developed to classify the weld defects. Neural networks use algorithms that learn functions, such as pattern recognition, creation of associations learning by training. The neural networks can process enormous amounts of data in a short period of time.[3]. Many researches have revealed the capability of Artificial Neural network for classifying weld defects. S. Sambath et al., [4] proposed a neural network based weld defect classification of pulse echo signals. The training algorithm used in that was Scaled conjugate gradient backpropagation. E P de Moura, et al.,[5] used a hierarchical and non hierarchical linear discriminator for classifying the weld defects. J. L. B. C. Veigav et al.,[6] has implemented a feed forward back propagation network with one hidden layer. Feed forward Multi layer perceptron ANNs with varying topologies were simulated in software and trained by Shaun W. Lawson et al [7]. C Shekhar N Shitole[8], developed neural-fuzzy classifier. Recurrent neural networks were used to classify natural language sentences [9] Yuwen Cao [10] used Feature selection based defect identification of ToFd images. But, This paper presents a resilient back propagation algorithm (RPROP) based artificial neural network, which is meant for classifying the defects of austenitic stainless steel weldments. In this work, the number of hidden layers are also increased from 0 to 6 and the classification performance is evaluated. Various performance functions like Mean Square Error function, Mean Absolute Error function, Mean square Error with regularization function and Sum squared Error performance functions were applied to find an optimum performance function for a better classification efficiency. The paper is structured as follows. Section 2 discusses the data acquisition module. Section 3 describes ANN modeling. Section 4 deals with the results and discussion. Conclusion and future work is described in section.

\section{DATA ACQUISITION MODULE}

Five austenitic stainless steel welds with the dimension of 200 x 200x $25 \mathrm{~mm}^{\wedge} 3$ are fabricated. The welds were of Double V Butt joint configuration and made by shielded metal arc welding process. Four defects such as lack of fusion, lack of penetration, slag and porosity have been artificially created into the four weldment respectively. One weldment has been fabricated without any defect. TOFD Experiment is conducted on these five weldments. Experiments are performed using $\mu$ TOFD of AEA Technology, UK to detect the defect. The photograph of the experimental set up is also shown in figure 1 .

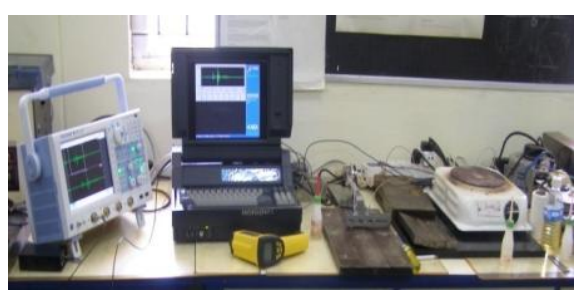

Figure 1. TOFD Experimental Set Up

A methodical approach has been adopted to ensure reliability of the experimental data by verifying it with radiography 
technique. The procedure adopted for testing includes calibration, job identification, visual inspection, scanning and defect identification. 40 ToFD A scan signals of each defect have been acquired.

\section{ANN MODELING}

\subsection{DENOISING}

The A scan signals are undergone an optimum denoising method, using discrete wavelet transform. An analysis have been done to find an optimum denoising method for the A scans of five different defects. The efficiency of the denoising methods are evaluated with their SNR values. Symlet 4 based denoising is found as the effective signal denoising algorithm for all the 5 different types of defected TOFD signals.[11].All the A scan signals are denoised using the above algorithm.

\subsection{FEATURE EXTRACTION}

Statistical features like mean, mode, median, standard deviation, maxima and minima are extracted from the denoised signals and are used as inputs to the neural network. Out of 40 signals of each defect,,30 signals were used for training the network and the remaining 10 are used for testing the network.

\subsection{NEURAL NETWORK}

Among the various types of neural networks, the multi-layer back-propagation neural network is suitable for the engineering applications.[12] The back-propagation network learns by back propagating the errors in the direction from output neurons to input neurons. The multi-layer network consists of an input layer, output layer and a number of hidden layers. The presence of hidden layers allows the network to represent and compute more complicated associations between patterns. Many researchers proved that the multi-layer back propagation with three layers can perform arbitrarily complex classification. $[13,14]$.Propagation of data takes place from input layer to the output layer. There is no connectivity between neurons in a layer. This type of neural network is trained using a process of supervised learning in which the network is presented with a series of matched input and output patterns and the connection strengths or weights of the connections automatically adjusted to decrease the difference between the actual and desired outputs. To begin with, patterns are presented to the network and a feedback signal which is equal to the difference between the desired and actual output is propagated backwards through the network for the adjustment of weights of the layers' connections according to the back propagation learning algorithm. trainrp is a network training function that updates weight and bias values according to the resilient backpropagation algorithm (RPROP). Learning is spread equally all over the entire network and the weights near the input layer have the equal chance to grow and learn as weights near the output layer. Rprop gives an efficient and transparent adaptation process. [15].Transfer functions convert a neural network layer's net input into its net output. In this network, the Transfer function of hidden layers is chosen as Symmetric sigmoid transfer function 'tansig' and Linear transfer function, 'purelin' for output layer. Back propagation weight/bias learning function is chosen as gradient descent with momentum weight/bias learning function. The network has been created with mean squared error performance function, mean absolute error function, Sum Square error performance function and mean squared error with regularization. The performances of the four performance functions are also evaluated in terms of the classification accuracy.

\section{RESULTS AND DISCUSSION}

Table 1. Classification accuracy in Percentage with respect to performance function

\begin{tabular}{|c|c|c|c|}
\hline $\begin{array}{c}\text { Performance } \\
\text { Function }\end{array}$ & $\begin{array}{l}\text { No of } \\
\text { hidden } \\
\text { layers }\end{array}$ & Training & Testing \\
\hline \multirow{7}{*}{ MSE } & 0 & 58.7 & 42 \\
\hline & 1 & 64.7 & 46 \\
\hline & 2 & 70 & 58 \\
\hline & 3 & 81.3 & 38 \\
\hline & 4 & 73.3 & 46 \\
\hline & 5 & 68.7 & 46 \\
\hline & 6 & 73 & 38 \\
\hline \multirow{7}{*}{ MAE } & 0 & 26 & 24 \\
\hline & 1 & 19.3 & 16 \\
\hline & 2 & 21.3 & 26 \\
\hline & 3 & 26.7 & 32 \\
\hline & 4 & 26.7 & 32 \\
\hline & 5 & 21.3 & 20 \\
\hline & 6 & 26.7 & 22 \\
\hline \multirow{7}{*}{ MSEREG } & 0 & 42.7 & 28 \\
\hline & 1 & 44 & 32 \\
\hline & 2 & 48 & 28 \\
\hline & 3 & 54.7 & 30 \\
\hline & 4 & 56 & 34 \\
\hline & 5 & 54.7 & 30 \\
\hline & 6 & 59.3 & 34 \\
\hline \multirow{7}{*}{ SSE } & 0 & 64 & 40 \\
\hline & 1 & 66 & 52 \\
\hline & 2 & 72 & 50 \\
\hline & 3 & 81.3 & 38 \\
\hline & 4 & 73.3 & 46 \\
\hline & 5 & 68.7 & 46 \\
\hline & 6 & 73.3 & 38 \\
\hline
\end{tabular}

To design an optimum network for classification of defects, the network architecture is varied in such a way of increasing the number of hidden layers. The change has been implemented only on number of hidden layers, whereas the training function, transfer function and learning function are kept unchanged for all the type of architecture. To know the 
impact of performance function on the network, MAE,MSE,SSE and MSEREG has been used one by one. The number of hidden layers is increased from 0 to 6 and for each change in the architecture, the network is trained with the above said statistical features and the network performance is studied with the defect classification performance. For For the hidden layers $0,1,2,3,4,5$ and 6 , the classification performance is tabulated according to the performance function. Table 1 shows the classification accuracy of all the performance functions with respect to the corresponding architecture. The MSE and SSE performed wee, when compared with MAE and MSEREG.
Table 2 and Table 3 show the the classification accuracy of all the defects with the performance functions MSE and SSE respectively. The best classification accuracy is achieved by hiddenlayers architecture with both MSE and SSE for the training data set. But, the testing performance is not quite promising compared to training performance.

Table 2. Classification accuracy in percentage with MSE

\begin{tabular}{|c|c|c|c|c|c|c|c|c|c|c|c|c|c|c|}
\hline \multirow[b]{3}{*}{ Defect Type } & \multicolumn{14}{|c|}{ No of hidden layers } \\
\hline & \multicolumn{2}{|c|}{$\mathbf{0}$} & \multicolumn{2}{|c|}{1} & \multicolumn{2}{|c|}{2} & \multicolumn{2}{|c|}{3} & \multicolumn{2}{|c|}{4} & \multicolumn{2}{|c|}{5} & \multicolumn{2}{|c|}{6} \\
\hline & Training & Testing & Training & Testing & Training & Testing & Training & Testing & Training & Testing & Training & Testing & Training & Testing \\
\hline Lack of penetration & 30 & 20 & 63.3 & 40 & 43.3 & 30 & 86.7 & 30 & 86.7 & 60 & 73.3 & 40 & 40 & 0 \\
\hline Lack of fusion & 53.3 & 10 & 66.7 & 50 & 73.3 & 60 & 63.3 & 20 & 46.7 & 20 & 33.3 & 10 & 70 & 30 \\
\hline No defect & 46.7 & 60 & 40 & 20 & 66.7 & 90 & 80 & 60 & 70 & 80 & 63.3 & 70 & 66.7 & 90 \\
\hline Porosity & 76.7 & 80 & 70 & 70 & 88.3 & 70 & 93.3 & 50 & 70 & 40 & 76.7 & 70 & 90 & 40 \\
\hline Slag & 86.7 & 40 & 83.3 & 50 & 83.3 & 40 & 83.3 & 30 & 93.3 & 30 & 96.7 & 40 & 100 & 30 \\
\hline Overall & 58.7 & 42 & 64.7 & 46 & 70 & 58 & 81.3 & 38 & 73.3 & 46 & 68.7 & 46 & 73 & 38 \\
\hline
\end{tabular}

Table 3. Classification accuracy in percentage with SSE

\begin{tabular}{|c|c|c|c|c|c|c|c|c|c|c|c|c|c|c|}
\hline \multirow[b]{3}{*}{ Defect Type } & \multicolumn{14}{|c|}{ No of hidden layers } \\
\hline & \multicolumn{2}{|c|}{$\mathbf{0}$} & \multicolumn{2}{|c|}{1} & \multicolumn{2}{|c|}{2} & \multicolumn{2}{|c|}{3} & \multicolumn{2}{|c|}{4} & \multicolumn{2}{|c|}{5} & \multicolumn{2}{|c|}{6} \\
\hline & Training & Testing & Training & Testing & Training & Testing & Training & Testing & Training & Testing & Training & Testing & Training & Testing \\
\hline Lack of penetration & 53.3 & 30 & 73.3 & 60 & 86.7 & 60 & 86.7 & 30 & 86.7 & 60 & 73.3 & 40 & 40 & 0 \\
\hline Lack of fusion & 40 & 20 & 43.3 & 20 & 40 & 0 & 63.3 & 20 & 46.7 & 20 & 33.3 & 10 & 70 & 30 \\
\hline No defect & 60 & 60 & 50 & 60 & 60 & 80 & 80 & 60 & 70 & 80 & 63.3 & 70 & 66.7 & 90 \\
\hline Porosity & 83.3 & 70 & 73.3 & 70 & 80 & 70 & 93.3 & 50 & 70 & 40 & 76.7 & 70 & 90 & 40 \\
\hline Slag & 83.3 & 20 & 90 & 50 & 93.3 & 40 & 83.3 & 30 & 93.3 & 30 & 96.7 & 40 & 100 & 39 \\
\hline Overall & 64 & 40 & 66 & 52 & 72 & 50 & 81.3 & 38 & 73.3 & 46 & 68.7 & 46 & 73.3 & 38 \\
\hline
\end{tabular}

\section{CONCLUSION}

Since, austenitic stainless steel is widely used for manufacturing reactor vessels ,the TOFD experiment is conducted on four defected austenitic stainless steel welds and one without any defect. For each weldment, 40 different A scans were acquired at different positions. To increase the quality of the signal, denoising was also performed on these A scans using Discrete wavelet transform. Six statistical features were extracted from the resultant denoised A scans. The features are given as input signals to the Feed forward Back propagation Neural Network, which was designed to classify the defects. The resilient training algorithm has been applied. TOFD images were used widely to identify defects, But, this novel approach used TOFD signals. For each defect, 30 signals have been given for training the network and 10 signals have been used for testing the network. Various types of architecture were designed to improve the classification performance of the network.. In order to improve the testing performance, the training algorithm can be changed. The classification accuracy can also be improved by extracting the multi scale features instead of single scale features. 


\section{ACKNOWLEDGMENTS}

The authors wish to thank the Scientists of Indira Gandhi Center for Atomic Research, Kalpakkam, Government of India for the technical support provided by them.

\section{REFERENCES}

[1] Elineudo P. Moura,Romeu R. Silva, and Joao Marcos A. Rebello Marcio H. S. Siqueira, Pattern recognition of Weld Defects in Preprocessed TOFD Signals Using Linear Classifiers, Journal of Nondestructive Evaluation, Vol. 23, No. 4, December 2004

[2] Shyamal Mondal,Dr.T.Sattar, An Overview of ToFD method and its mathematical model, NDT.net-April 2000, Vol 5.No.4

[3] S. Haykin:Neural Networks, A Comprehensive Foundation.1994, Macmillian College Publishing

[4] S. Sambath , P. Nagaraj, N. Selvakumar: Automatic Defect Classification in Ultrasonic NDT Using Artificial Intelligence. Journal of Nondestrucive Evaluation (2011) 30: 20-28

[5] E P de Moura, M H S Siqueira, R R da Silva, J M A Rebello and L P Calôba: Welding defect pattern recognition in TOFD signal Part 1. Linear classifier.Insight Vol 47 No 12 December 2005

[6] J. L. B. C. Veiga, A. A. de Carvalho,I. C. da Silva,and J. M. A. Rebello:The Use of Artificial Neural Network in the Classification of Pulse-Echo and TOFD Ultra-Sonic Signals. October-December 2005 ABC394 M

[7] Shaun W. Lawson and Graham A. Parker: Automatic detection of defects in industrial ultrasound images using a neural network.
[8] C'Shekhar N Shitole, O Zahran and W Al-Nuaimy, Combining fuzzy logic and neural networks in classification of weld defects using ultrasonic time-offlight diffraction, NDT 2006, the 45th Annual British Conference on NDT, Stratford-upon-Avon, UK, September 2006

[9] Sergio Roa and Fernando: Classification of Natural Language Sentences using Neural Networks, FLAIRS 2003

[10] YuwenCao, HaijiangZhu, PingYang: Ultrasonic Time of Flight Diffraction Defect Recognition Based on Edge Detection, Biomedical Engineering and Computer Science (ICBECS), 2010 International Conference, 2325 April, 2010 Wuhan, China

[11] S.LalithaKumari, B.SheelaRani, B.Venkatraman, Wavelet Transform based De-noising of ToFD signals of austenitic stainless steel welds, CiiT International Journal of_Digital Signal Processing, Oct 2011

[12] M.S.Obaidat,M. A. Suhail and B. Sadoun : An intelligent simulation methodology to characterize defects in materials. Information Sciences Volume 137, Issues 1-4, September 2001,pp33-41

[13] Amitava Roy, P. Barat and Swapan Kumar De :Material classification through neural networks. Ultrasonics ,Volume 33, Issue 3, May 1995, pp 175-180

[14] Karray and de Silva : Soft Computing and Intelligent Systems Design.Addison Wesley September 27, 2004

[15] Martin Riedmiller, Heinrich Braun:A Direct Adaptive Method for Faster Backpropagation Learning, The RPROP Algorithm. (1993) IEEE International Conference On Neural Networks 